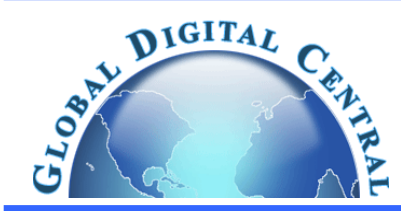

Frontiers in Heat and Mass Transfer

Available at www.ThermalFluidsCentral.org

\title{
COMBINED EFFECTS OF HALL, JOULE HEATING AND THERMAL DIFFUSION ON MIXED CONVECTION FLOW IN A VERTICAL CHANNEL SATURATED WITH COUPLE STRESS FLUID
}

\author{
K. Kaladhar ${ }^{\mathrm{a}, *}$ and D. Srinivasacharya ${ }^{\mathrm{b}}$ \\ a Department of Mathematics, National Institute of Technology Puducherry, Karaikal-609605, India \\ ${ }^{\mathrm{b}}$ Department of Mathematics, National Institute of Technology, Warangal-506004, India
}

\begin{abstract}
This present investigation carried out the effects of Hall current, Joule heating and the thermal diffusion on mixed convection flow of electrically conducting couple stress fluid in a vertical channel saturated with porous medium. The final system of ordinary differential equations are obtained from the governing non-linear partial differential equations by using the similarity transformations. Homotopy Analysis Method has been used to solve the non-linear system. The average residue errors of the HAM solutions are presented through graphs. The influence of the emerging parameters (Hall, Soret, magnetic and the couple stress parameters) on velocity, temperature and concentration profiles are presented through plots. Finally heat and mass transfer rates at both the plates are presented in tabular form.

Keywords: Mixed convection, Joule heating effect, Soret effect, Couple stress fluid, Hall effect, HAM.
\end{abstract}

\section{INTRODUCTION}

Combined heat and mass transfer in mixed convection through a vertical channel flow has considerable importance in many applications. Much of the early literature of theoretical investigations and their applications on mixed convection flow between parallel plates with heat and mass transfer have been reported in Boulama and Galanis (2004). In their study, the analytical expressions for velocity, temperature, and mass fraction profiles are obtained and the conditions of flow reversal are analyzed for both the cases UHF and UHT. Fattahi et al. (2012) applied the lattice boltzmann technique to study the nature of free convection flow of nano fluids in a square cavity. Huelsz and Rechtman (2013) also applied the lattice boltzmann technique for Heat transfer in an inclined square cavity due to natural convection of air in the laminar regime as both the Rayleigh number and the inclination angle of the cavity change, in which the range of the inclination angle covers a whole revolution. Martyushev and Sheremet (2014) analyzed the Conjugate natural convection flow with surface radiation in an air filled in square cavity enclosure having heat conducting solid walls of finite thickness and a local heat source in conditions of convective heat exchange with an environment. Most recently, Hasnain et al. (2015) presented the effects of porosity and mixed convection on MHD two phase fluid flow in an inclined channel.

In recent years, considerable attention has been devoted to Hall current effect due to importance in many astrophysical and geophysical situations as well as in engineering problems such as centrifugal machines, constructions of turbines and Hall accelerators, induction flow meters, MHD flows, planetary, solar dynamic problems and the studies which can be helpful in prediction of the effects of magnetic intrusions. Also the effect Joule heating is usually characterized by the product of the magnetic parameter and Brinkman number, and it has a very important role in geophysical flow and in nuclear engineering (Rahman et al. (2011)). Most recently, Zhang et al. (2015) presented the The Joule heating effects on natural convection flow of participating magnetohydrodynamics under different levels of thermal radiation considering the Hall effect in a cavity. After detailed analysis, Zhang et al. (2015) were found that the Joule heating has notable effect on fluid flow and heat transfer in the cavity and hence Joule heating cannot be neglected in certain range of parameters.

Many fluids in technical processes exhibit non- Newtonian behavior since the conventional Newtonian fluids are not specifically explains the properties of real fluids. Among all such non- Newtonian fluids, Stokes (1966) introduced the couple stress fluids, which have different features such as non-symmetric stress tensor, body couples and couple stresses. These fluids are able to describe various types of blood, suspension fluids, lubricants etc. Later, Stokes (1984) was reported a review of couple stress fluid dynamics. Then the basic properties, applications and the past literature of the couple stress fluids in different geometries was addressed by Makinde and Eegunjobi (2013). Most recently, Turkyilmazoglu (2014)

${ }^{*}$ Corresponding author. Email: kkr.nitpy@gmail.com 
presented the exact solutions for two-dimensional laminar flow over a continuously stretching or shrinking sheet in an electrically conducting quiescent couple stress fluid, in which the exact solutions for both flow and temperature fields under a boundary layer approach are targeted.

The influence of temperature gradient for the mass diffusion is called the thermo-diffusion or soret effect. Soret effect has considerable attention in the flow regime when there exist a large density differences and this will accelerate in presence of magnetic field. The applications and early literature can be seen in the work of Afify (2009). Recently, Mahmoud and Megahed (2013) presented the thermal radiation and cross diffusion effects on mixed convection flow of a non-Newtonian power-law fluids over a vertical surface embedded in a porous medium, the analysis shown that the local Nusselt number increases as the suction parameter or Soret number increases and decreases with increasing the injection parameter or Dufour number in both pseudo-plastic and dilatant fluids. Most recently, Srinivasacharya et al. (2015) studied the nature of soret and dufour effects on mixed convection along a vertical wavy surface in a porous medium with variable properties in which the viscosity and thermal conductivity of the fluid are assumed to be varying with respect to temperature.

In this paper, the mixed convection flow of a couple stress fluid through a vertical channel saturated with porous medium is investigated in presence of thermal diffusion, Hall current and Joule effect. The above literature clearly shows that the physical significance of the effects of Hall current under the uniform magnetic field, joule heating and thermal diffusion effect in non- Newtonian fluids in a channel. The survey clearly shows that the combined effects on couple stress fluid through vertical channel in porous medium has not been presented else where. In view of applications and importance, the authors are motivated to take this problem. The Homotopy Analysis Method (HAM) is employed to solve this nonlinear problem. The HAM was proposed by Liao (2003). The method of approach, recent developments of the HAM can be seen in Liao (2010); Srinivasacharya and Kaladhar (2012). Detailed description of HAM has been reported by Liao (2014). In this work, optimal values of the auxiliary parameters were presented in brief, also the convergence of the resulting series solution is explained. Finally the influence of various flow parameters on velocities, temperature and concentration is discussed.

\section{FORMULATION OF THE PROBLEM}

Consider an electrically conducting incompressible couple stress fluid flow in a vertical channel saturated with porous medium. Cartesian coordinate system is chosen in such a way that $x$-axis be taken along vertically upward direction through the central line of the channel, $y$ makes right angle to vertical plates and both the plates are extended infinitely in $x$ and $z$ directions. The vertical channel plates are placed at $y= \pm d$. Uniform magnetic field is applied perpendicular to the plates (flow direction), which causes the Hall and Joule heating effects in the flow regime. The additional force in the $z$-direction is generated due to the effect of Hall current, which gives rise to a cross flow therefore the flow becomes three dimensional. The induced magnetic field can be neglected by assuming that the magnetic Reynolds number is very small in study flow in comparison with the applied magnetic field. Buoyancy forces and the uniform pressure gradient in $x$ direction leads to the mixed convection flow. The coordinate system and configuration of the flow is presented in Figure 1. In addition, all the fluid properties are assumed to be constant except the density in the buoyancy term of the balance of momentum equation. With the above presumptions, the governing equations for the incompressible steady flow of couple stress fluid with MHD approximations are

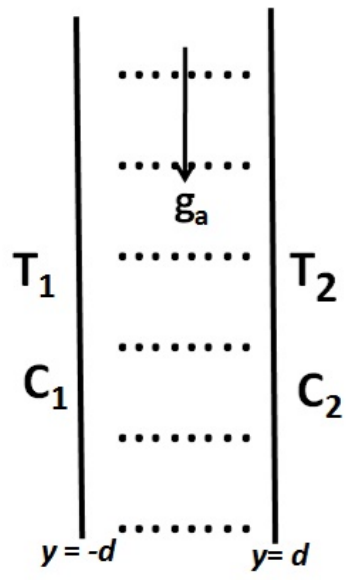

Figure 1: Physical model and coordinate system.

$$
\begin{gathered}
\rho v \frac{\partial u}{\partial y}=\mu \frac{\partial^{2} u}{\partial y^{2}}+\rho g_{a} \beta_{T}\left(T-T_{1}\right)+\rho g_{a} \beta_{C}\left(C-C_{1}\right) \\
-\eta_{1} \frac{\partial^{4} u}{\partial y^{4}}-\frac{\mu}{K_{f}} u-\frac{\sigma B_{0}^{2}}{1+\beta_{h}^{2}}\left(u+\beta_{h} w\right)-\frac{d p}{d x} \\
\rho v \frac{\partial w}{\partial y}=\mu \frac{\partial^{2} w}{\partial y^{2}}+\frac{\sigma B_{0}^{2}}{1+\beta_{h}^{2}}\left(\beta_{h} u-w\right)-\eta_{1} \frac{\partial^{4} w}{\partial y^{4}}-\frac{\mu}{K_{f}} w \\
\rho C_{p} v \frac{\partial T}{\partial y}=K_{f} \frac{\partial^{2} T}{\partial y^{2}}+2 \mu\left[\left(\frac{\partial u}{\partial y}\right)^{2}+\left(\frac{\partial w}{\partial y}\right)^{2}\right] \\
+\eta_{1}\left[\left(\frac{\partial^{2} u}{\partial y^{2}}\right)^{2}+\left(\frac{\partial^{2} w}{\partial y^{2}}\right)^{2}\right]+\frac{\sigma B_{0}^{2}}{1+\beta_{h}^{2}}\left(u^{2}+w^{2}\right) \\
v \frac{\partial C}{\partial y}=D \frac{\partial^{2} C}{\partial y^{2}}+\frac{D K_{f}}{T_{m}} \frac{\partial^{2} T}{\partial y^{2}}
\end{gathered}
$$

with

$$
\begin{gathered}
u=0 \quad \text { at } y= \pm d \\
u_{y y}=0 \quad \text { at } y= \pm d \\
T=T_{1}, \quad \text { at } y=-d, \quad T=T_{2}, \quad \text { at } \quad y=d \\
C=C_{1}, \quad \text { at } y=-d, \quad C=C_{2}, \quad \text { at } \quad y=d
\end{gathered}
$$

where the boundary condition (6b) imply that the couple stresses are zero at the plate surfaces.

The velocity components in $x-, y-$ and $z-$ are $u, v, w$ respectively, $g_{a}$ is the acceleration due to gravity, $\rho$ is the density, $C_{p}$ is the specific heat, $\mu$ is the coefficient of viscosity, $\beta_{h}$ is the Hall parameter, $\beta_{T}$ is the coefficient of thermal expansion, $\beta_{C}$ is the coefficient of solutal expansion, $\sigma$ is the electric conductivity of the fluid, $B_{0}$ is the uniform magnetic field, $D$ is the mass diffusivity, $K_{f}$ is the coefficient of thermal conductivity, $T_{m}$ is the mean fluid temperature and $\eta_{1}$ is the additional viscosity coefficient which specifies the character of couple-stresses in the fluid. The last terms of (4), (5) represents the joule heating and tharmal diffusions respectively. Equation (1) implies that the velocity in $y$ direction $v$ is constant i.e. $v=v_{0}$.

Introducing the following dimensionless variables

$$
\begin{array}{r}
y=\eta d, \quad u=u_{0} f, \quad w=u_{0} g, T-T_{1}=\left(T_{2}-T_{1}\right) \theta \\
C-C_{1}=\left(C_{2}-C_{1}\right) \phi, \quad p=\frac{\mu u_{0}}{d^{2}} P
\end{array}
$$
tions as

in equations (2) - (5), we obtain the governing dimensionless equa-

$$
v_{y}=0
$$




$$
\begin{gathered}
S^{2} f^{(i v)}-\frac{G r_{T}}{R e} \theta-\frac{G r_{C}}{R e} \phi-f^{\prime \prime}+R f^{\prime}+\frac{1}{D a} f \\
+\frac{H a^{2}}{1+\beta_{h}^{2}}\left(f+\beta_{h} g\right)+A=0 \\
S^{2} g^{(i v)}-g^{\prime \prime}+R g^{\prime}-\frac{H a^{2}}{1+\beta_{h}^{2}}\left(\beta_{h} f-g\right)+\frac{1}{D a} g=0 \\
\theta^{\prime \prime}-\operatorname{RPr} \theta^{\prime}+2 B r\left[\left(f^{\prime}\right)^{2}+\left(g^{\prime}\right)^{2}\right]+\frac{J}{1+\beta_{h}^{2}}\left(f^{2}+g^{2}\right) \\
+S^{2} B r\left[\left(f^{\prime \prime}\right)^{2}+\left(g^{\prime \prime}\right)^{2}\right]=0 \\
\phi^{\prime \prime}-R S c \phi^{\prime}+S c S r \theta^{\prime \prime}=0
\end{gathered}
$$

with

$$
\begin{array}{r}
f=g=f^{\prime \prime}=g^{\prime \prime}=\theta=\phi=0 \quad \text { at } \quad \eta=-1 \\
f=g=f^{\prime \prime}=g^{\prime \prime}=0 \text { and } \theta=\phi=1 \text { at } \eta=1
\end{array}
$$

where the primes denote differentiation with respect to $\eta, S=\frac{1}{d} \sqrt{\frac{\eta_{1}}{\mu}}$ is the couple stress parameter, $R=\frac{\rho v_{0} d}{\mu}$ is the suction/injuction parameter, $G r_{T}=\frac{\rho^{2} g \beta_{T} d^{3}}{\mu^{2}}\left(T_{2}-T_{1}\right)$ is the energy Grashof number, $G r_{C}=$ $\frac{\rho^{2} g \beta_{C} d^{3}}{\mu^{2}}\left(C_{2}-C_{1}\right)$ is the thermal Grashof number, $B r=\frac{\mu u_{0}^{2}}{K_{f}\left(T_{2}-T_{1}\right)}$ is the Brinkman number, $S_{r}=\frac{D K_{T}\left(T_{2}-T_{1}\right)}{\nu T_{m}\left(C_{2}-C_{1}\right)}$ is the Soret number, $D a=\frac{u_{0} K_{f}}{d^{2}}$ is the darcy number, $H a=B_{0} d \sqrt{\frac{\sigma}{\mu}}$ is the magnetic parameter, $R e=\frac{\rho v_{0} d}{\mu}$ is the Reynolds number, $\operatorname{Pr}=\frac{\mu C_{p}}{K_{f}}$ is the Prandtl number, $u_{0}$ is the entrance velocity, $A=\frac{d P}{d x}$ is the constant pressure gradient, $S c=\frac{\nu}{D}$ is the Schmidt number and $J=H a^{2} B r$ is the Joule heating parameter.

The physical quantities of interest in this problem are the skin friction coefficient, the Nusselt number and the Sherwood number. The shearing stress, heat, mass fluxes at the vertical plate surfaces can be obtained from

$$
\left.\left.\tau_{w}=\left.\mu \frac{\partial u}{\partial y}\right|_{y= \pm d} ; q_{w}=-K_{f} \frac{\partial T}{\partial y}\right]_{y= \pm d}, q_{m}=-D \frac{\partial C}{\partial y}\right]_{y= \pm d}
$$

The non-dimensional shear stress $C_{f}=\frac{\tau_{w}}{\rho u_{0}^{2}}$, the Nusselt number $N u=\frac{q_{w} d}{K_{f}\left(T_{2}-T_{1}\right)}$ and the Sherwood number $S h=\frac{q_{m} d}{D\left(C_{2}-C_{1}\right)}$ are given by

$$
\begin{array}{r}
R e C_{f_{1}}=2 f^{\prime}(-1) ; \quad R e C_{f_{2}}=2 f^{\prime}(1), N u_{1,2}=-\left.\theta^{\prime}(\eta)\right|_{\eta=-1,1} \\
S h_{1,2}=-\left.\phi^{\prime}(\eta)\right|_{\eta=-1,1} ;
\end{array}
$$

Effect of the various parameters involved in the investigation on these coefficients is discussed in the following section.

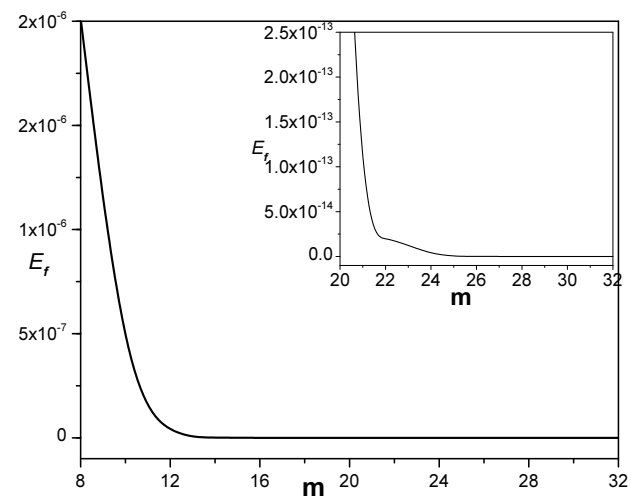

Figure 2: The average residual error of $f(\eta)$

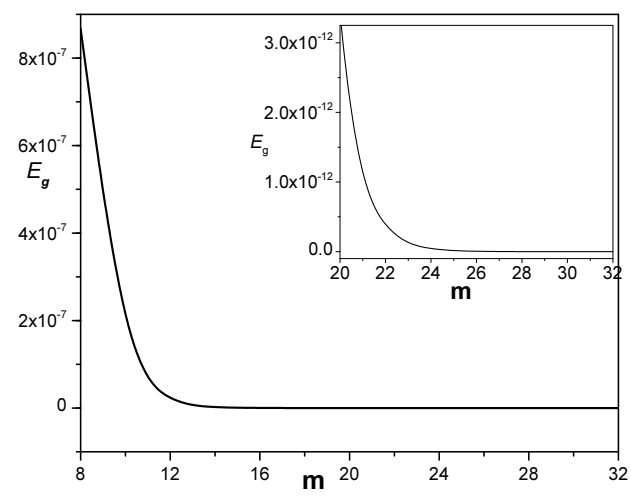

Figure 3: The average residual error of $g(\eta)$

\section{THE HAM SOLUTION OF THE PROBLEM}

The initial approximations of the velocities $(f(\eta), g(\eta))$, temperature $\theta(\eta)$ and the concentration $\phi(\eta)$ are chosen for HAM solutions as:

$$
f_{0}(\eta)=0, g_{0}(\eta)=0, \theta_{0}(\eta)=\frac{1+\eta}{2}, \phi_{0}(\eta)=\frac{1+\eta}{2}
$$

with the auxiliary linear operators are

$$
L_{1}=\frac{\partial^{4}}{\partial \eta^{4}} \quad \text { and } \quad L_{2}=\frac{\partial^{2}}{\partial \eta^{2}}
$$

such that

$$
L_{1}\left(c_{1} \eta^{3}+c_{2} \eta^{2}+c_{3} \eta+c_{4}\right)=0, \quad L_{2}\left(c_{5} \eta+c_{6}\right)=0
$$

where $c_{1}, c_{2}, \ldots, c_{6}$ are constants. The non-zero convergence control parameters $h_{1}, h_{2}, h_{3}$ and $h_{4}$ are introduced in zeroth-order deformations.

For HAM solutions the zeroth-order deformations; non-linear operators $N_{1}, N_{2}, N_{3}$ and $N_{4}$; average residual errors of $f, g, \theta$ and $\phi$ are considered as explained in the work of Srinivasacharya and Kaladhar (2012).

For the optimal values of the auxiliary parameters $h_{1}, h_{2}, h_{3}$ and $h_{4}, h$-curves are plotted by taking the values of the parameters as $G r_{T}=$ $4, G r_{C}=4, H a=5, \beta_{h}=2, D a=0.5, R e=2, \operatorname{Pr}=0.71, S=$ 


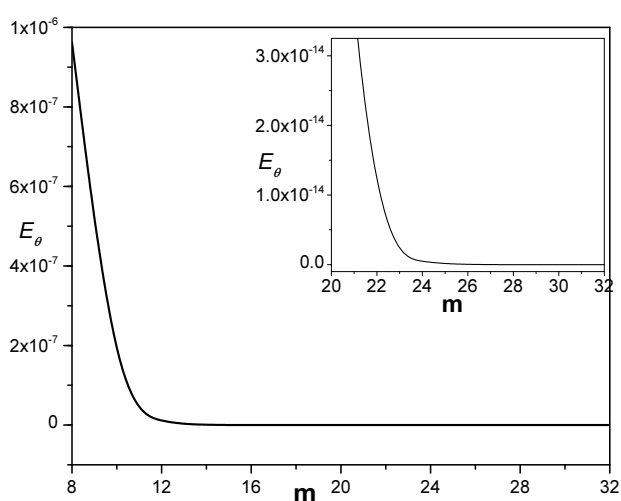

Figure 4: The average residual error of $\theta(\eta)$

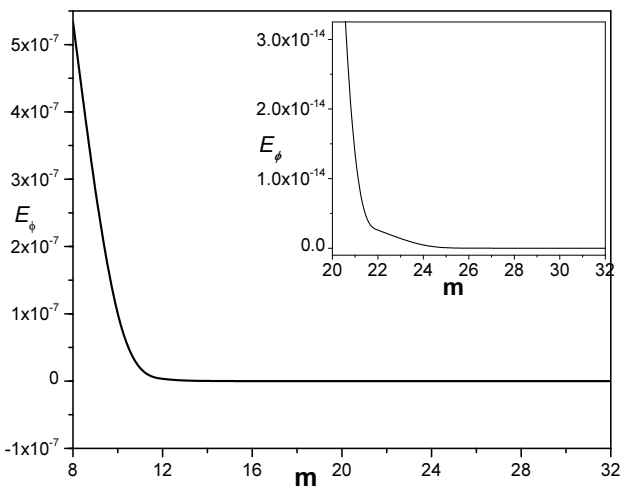

Figure 5: The average residual error of $\phi(\eta)$

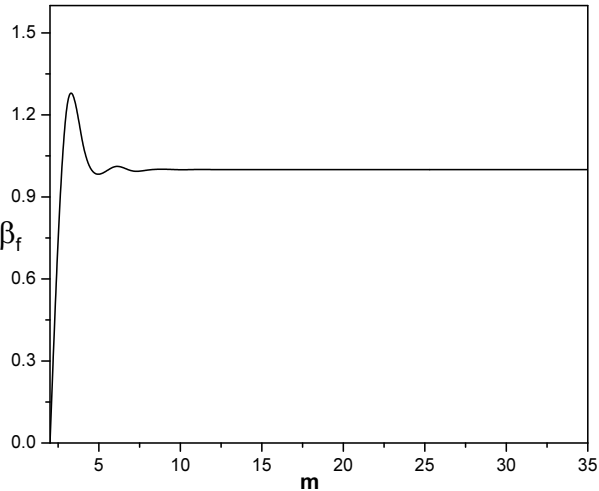

Figure 6: The ratio $\beta_{f}$ from the theorem to reveal the convergence of the HAM solutions

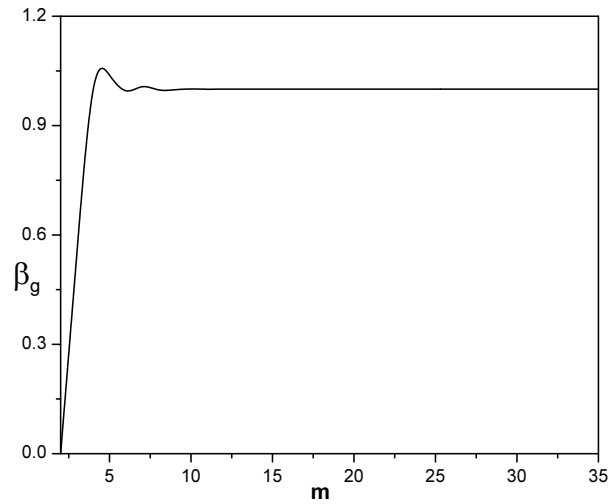

Figure 7: The ratio $\beta_{g}$ from the theorem to reveal the convergence of the HAM solutions

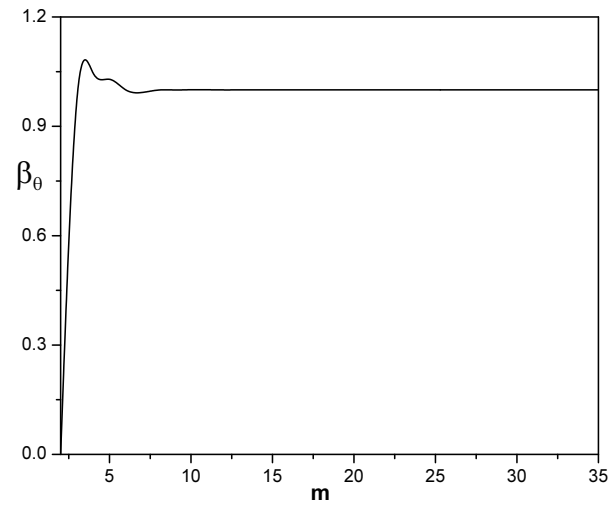

Figure 8: The ratio $\beta_{\theta}$ from the theorem to reveal the convergence of the HAM solutions

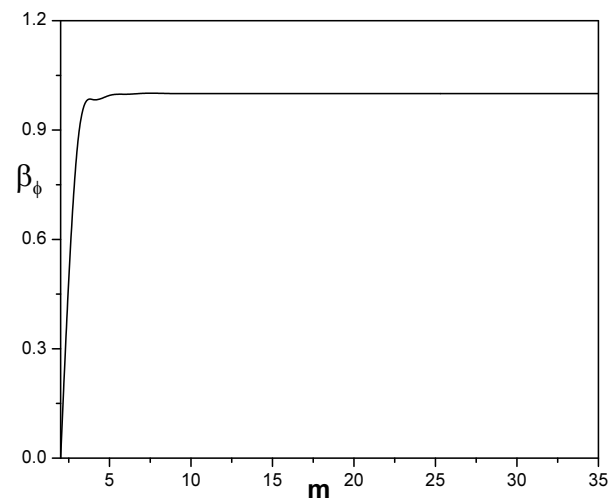

Figure 9: The ratio $\beta_{\phi}$ from the theorem to reveal the convergence of the HAM solutions 


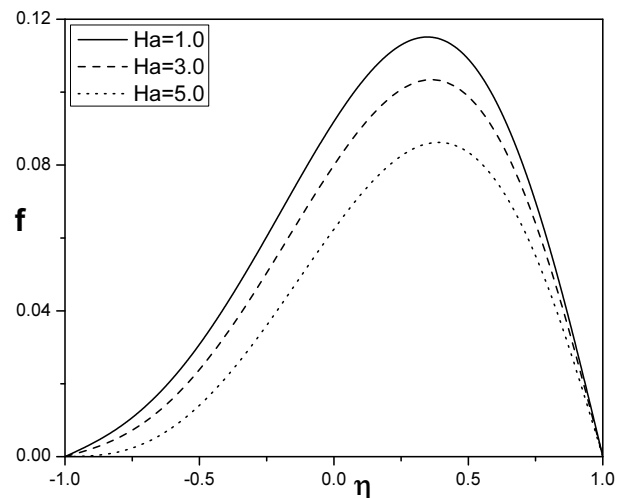

Figure 10: Magnetic parameter effect on $f(\eta)$ when $\beta_{h}=2, S=$ $0.5, \mathrm{Ha}=5, \mathrm{Sr}=2.0, \mathrm{Da}=0.5$

$0.5, S c=0.22, B r=0.1, J=1, A=0.5, S r=1$. The permissible values of $h_{1}, h_{2}, h_{3}$ and $h_{4}$ are obtained as -0.666044, -0.404893, $0.805937,-1.0981$ respectively with the average residue errors (explained in Srinivasacharya and Kaladhar (2012)) shown in Figs. 2-5. Finally the total average residual errors are presented in Table 1 at different order of approximations and the convergence of the series solutions are shown in Table 2.

In order to pursue the convergence of the HAM solutions to the exact ones, the graphs for the ratio (following the recent work of Turkyilmazoglu $(2011 a, b))$

$$
\begin{array}{ll}
\beta_{f}=\left|\frac{f_{m}(\eta)}{f_{m-1}(\eta)}\right|, \quad \beta_{g}=\left|\frac{g_{m}(\eta)}{g_{m-1}(\eta)}\right| \\
\beta_{\theta}=\left|\frac{\theta_{m}(\eta)}{\theta_{m-1}(\eta)}\right|, \quad \beta_{\phi}=\left|\frac{\phi_{m}(\eta)}{\phi_{m-1}(\eta)}\right|
\end{array}
$$

against the number of terms $m$ in the homotopy series is presented in Figs. 6-9. Figures strongly indicate that a finite limit of $\beta$ will be attained in the limit of $m \rightarrow \infty$, which will remain less than unity. The velocity and temperature solutions seem to converge in an oscillatory manner requiring more terms in the homotopy series. Thus, the convergence to the exact solution is assured by the HAM method.

\section{DISCUSSION OF RESULTS}

In absence of Hartmann number $H a$, suction/injuction parameter $R$, Darcy effect and the Buoyancy ratios $\left(G r_{T} / R e\right.$ and $\left.G r_{C} / R e\right)$, Eqn. (8) reduces to the equation of motion for the flow between parallel plates given in text book (Stokes (1966), page no. 44). Analytical solution of that equation with type $A$ conditions and HAM solution at different $S$ are shown in Table 3 . The comparisons are found to be in a very good agreement. Therefore, the HAM code can be used with great confidence to study the problem considered in this paper.

The profiles of velocities $(f(\eta), g(\eta))$, temperature $(\theta(\eta))$ and concentration $(\phi(\eta))$ are computed and presented through plots in Figs. 10 to 29 with different values of $H a, \beta_{h}, S r, S$. Computations were carried out by fixing the parameters $G r_{T}=4, G r_{C}=4, R e=2, \operatorname{Pr}=0.71, S c=$ $0.22, B r=0.1, J=1, A=0.5$ to analyze the effects of the emerging parameters $H a, \beta_{h}, S r$ and $S$.

Figure 10 presents the influence of the Hartman number $\mathrm{Ha}$ on $f(\eta)$. It is clear that increase in the parameter $H a$ leads to decrease the flow velocity $f(\eta)$. It is obvious that the nature of the transverse magnetic field normal to the flow direction has an affinity to create drag (nothing but Lorentz force), which leads to resist the flow. Therefore increase

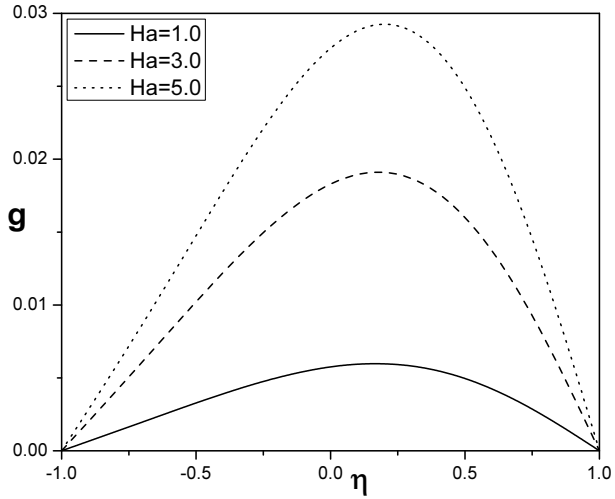

Figure 11: Magnetic parameter effect on $g(\eta)$ when $\beta_{h}=2, S=$ $0.5, \mathrm{Ha}=5, \mathrm{Sr}=2.0, \mathrm{Da}=0.5$

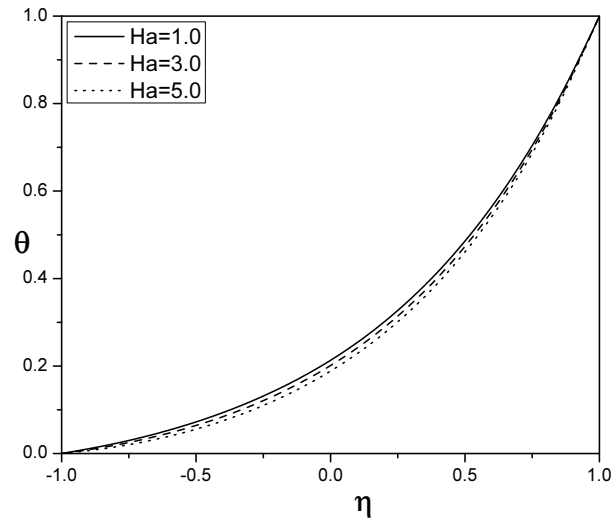

Figure 12: Magnetic parameter effect on $\theta(\eta)$ when $\beta_{h}=2, S=$ $0.5, \mathrm{Ha}=5, \mathrm{Sr}=2.0, \mathrm{Da}=0.5$

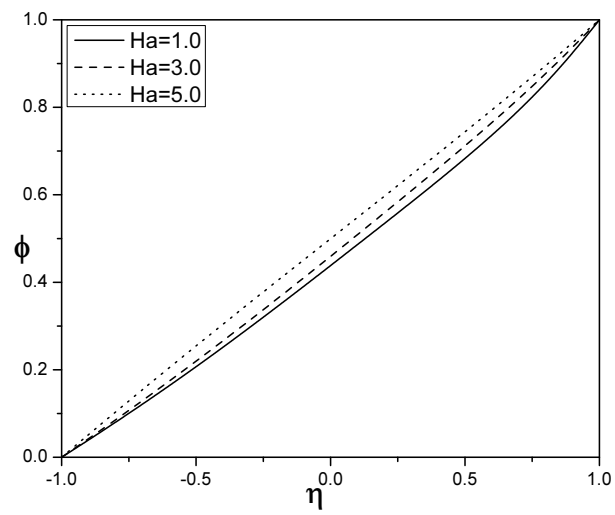

Figure 13: Magnetic parameter effect on $\phi(\eta)$ when $\beta_{h}=2, S=$ $0.5, \mathrm{Ha}=5, \mathrm{Sr}=2.0, \mathrm{Da}=0.5$ 


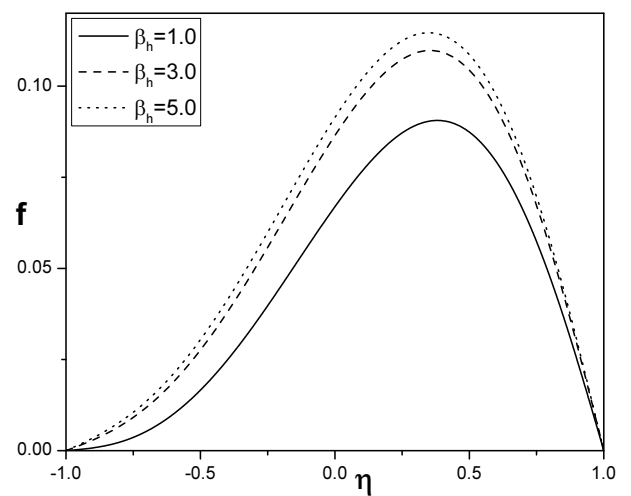

Figure 14: Hall effect on $f(\eta)$ when $S=0.5, \mathrm{Ha}=5, \mathrm{Sr}=2.0, \mathrm{Da}=$ 0.5

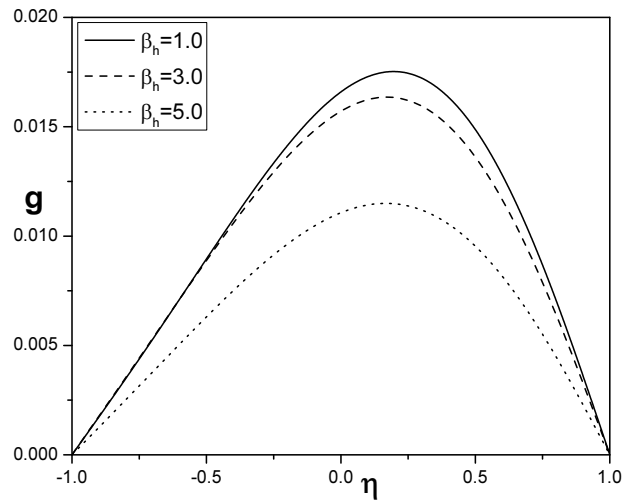

Figure 15: Hall effect on $g(\eta)$ when $S=0.5, H a=5, S r=2.0, D a=$ 0.5

in magnetic parameter influences to decrease the flow velocity. Figure 11 shows that the effect of $\mathrm{Ha}$ on the induced flow velocity $g(\eta)$. It is noticed that the cross flow velocity increases as the magnetic parameter increases. Figure 12 shows the influence of $\mathrm{Ha}$ on temperature profile. It can be noticed from this figure that as $\mathrm{Ha}$ increases the temperature of the flow decreases. The effect of magnetic parameter on $\phi(\eta)$ is revealed in Fig. 13. From this figure it is clear that increase in $\mathrm{Ha}$ leads to increase in concentration. As discussed above, the Lorentz force creates resistance in the fluid which leads to the friction between the fluid layers. Hence the temperature of the fluid decreases and concentration increases.

The variation of velocity components $(f(\eta)$ and $g(\eta))$, temperature $\theta(\eta)$ and concentration $\phi(\eta)$ with $\beta_{h}$ are shown in Figs.14 to 17 . We see that the dimensionless velocity component $f(\eta)$ increases with an increase in the Hall parameter. It can be seen from Fig. 15 that the induced velocity decreases as $\beta_{h}$ increases. This is due to the inclusion of Hall parameter decreases the resistive force imposed by the magnetic field due to its effect in reducing the effective conductivity. The effect of Hall parameter on temperature profile is shown in Fig. 16. It is clear from this figure that the non-dimensional temperature profile increases with the increase of Hall parameter. Literature shows that the hall effect is not significant on temperature profile, but in this study the effect of $\beta_{h}$ on temperature is markable because of joule heating and dissipation effects. Finally the influence of Hall number on concentration is shown in Fig. 17. It is

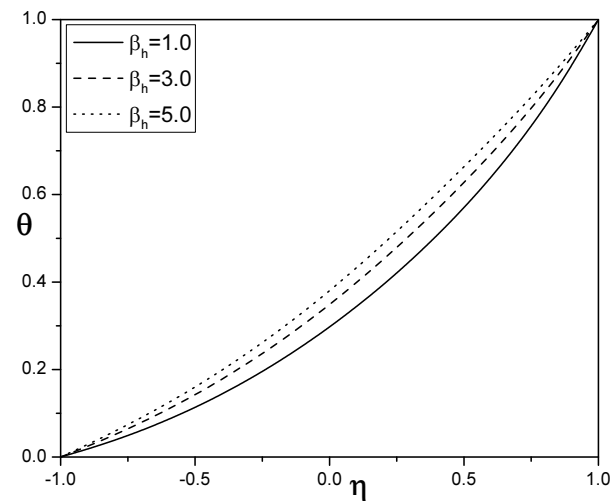

Figure 16: Hall effect on $\theta(\eta)$ when $S=0.5, H a=5, S r=2.0, D a=$ 0.5

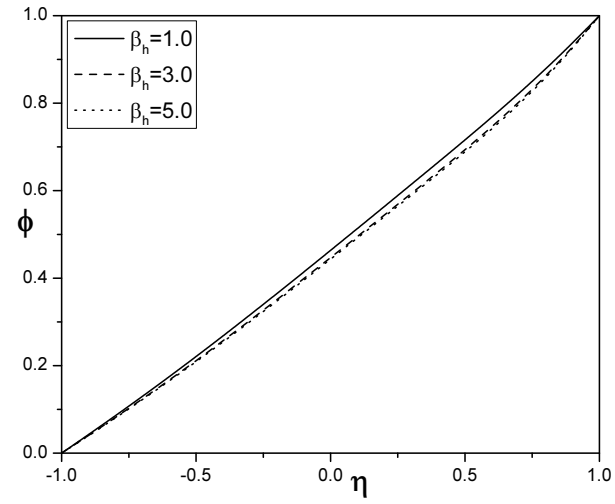

Figure 17: Hall effect on $\phi(\eta)$ when $S=0.5, H a=5, S r=2.0, D a=$ 0.5

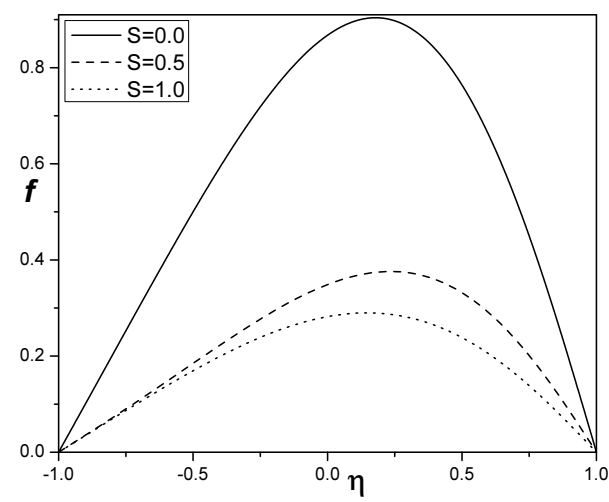

Figure 18: Effect of $S$ on $f$ at $H a=2, \beta_{h}=2, S r=2.0, D a=0.5$ 


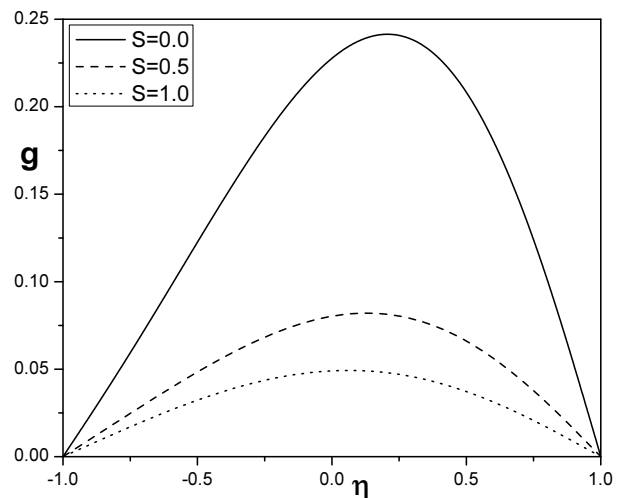

Figure 19: Effect of $S$ on $g$ at $H a=2, \beta_{h}=2, S r=2.0, D a=0.5$

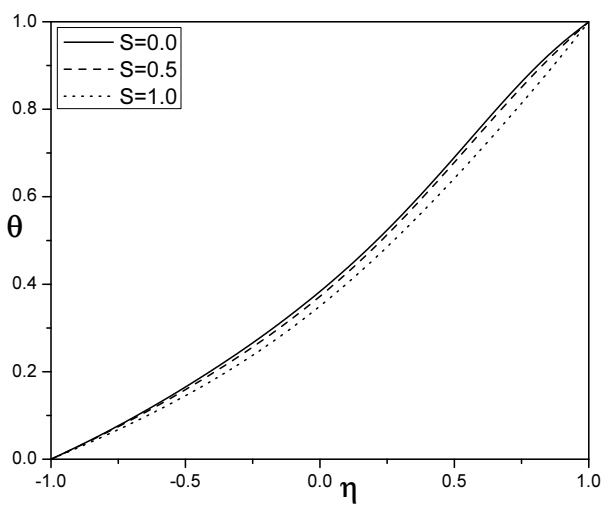

Figure 20: Effect of $S$ on $\theta$ at $H a=2, \beta_{h}=2, S r=2.0, D a=0.5$

observed from the figure that the dimensionless concentration decreases with an increase in Hall parameter. This is due to the fact that increase in temperature suppresses the concentration.

Influence of couple stress fluid on velocities, temperature and concentration are shown in Figs. 18 to 21 . The flow velocity $f(\eta)$, the induced flow in the z-direction $f(\eta)$ and the non dimensional temperature profile decreases with the increase in $S$. Since increase in couple stress parameter leads to the increase in the stress between all the coupled fluid particles, this leads to the decrease in the velocities and the temperature profile. It can be seen from fig. 21 that the increase in $S$ leads to increase in $\phi(\eta)$. It is observed that the newtonian fluid velocity is more than that of the non-Newtonian fluid (Couple stress fluid).

Figures 22-23 reveal that the higher values of soret number $\mathrm{Sr}$ decreases the velocities. Since the values of $S r$ increases due to either decrease in the temperature difference or increase in the concentration difference. Therefore the velocity profiles decreases with the increase of the Soret number i.e., the lowest peak of the reverse flow velocity compatible with the highest Soret parameter. Fig. 24 shows the influence of $S r$ on temperature profile. It can seen from this figure that the temperature decreases with the higher values of $\mathrm{Sr}$. this is due to the flow heats vigorously with the decrease of the Soret parameter. Figure 25 explains the nature of the concentration profile for different values of $\mathrm{Sr}$. It is seen that the concentration of the fluid increases with the increase of Soret number. This is because of temperature gradients contribution to diffusion of the species. The present analysis shows that the flow field is

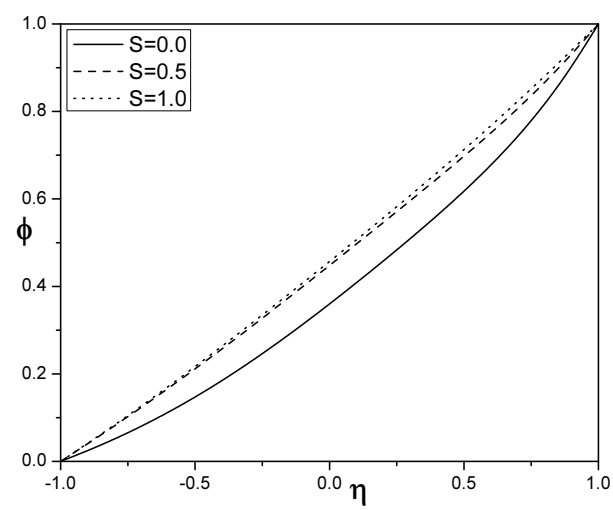

Figure 21: Effect of $S$ on $\phi$ at $H a=2, \beta_{h}=2, S r=2.0, D a=0.5$

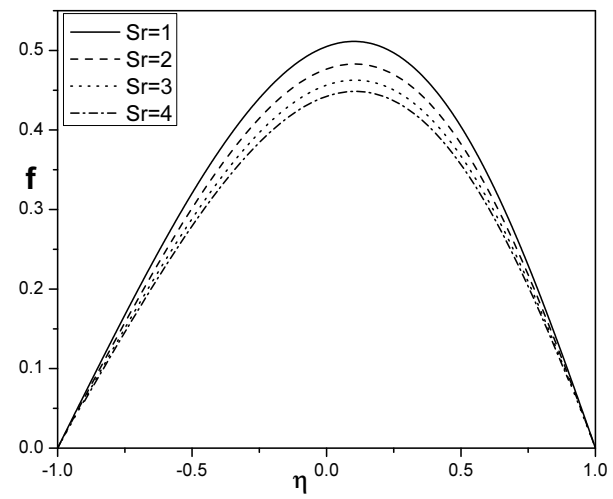

Figure 22: Effect of $S r$ on $f$ at $S=1, H a=2, \beta_{h}=2.0, D a=0.5$

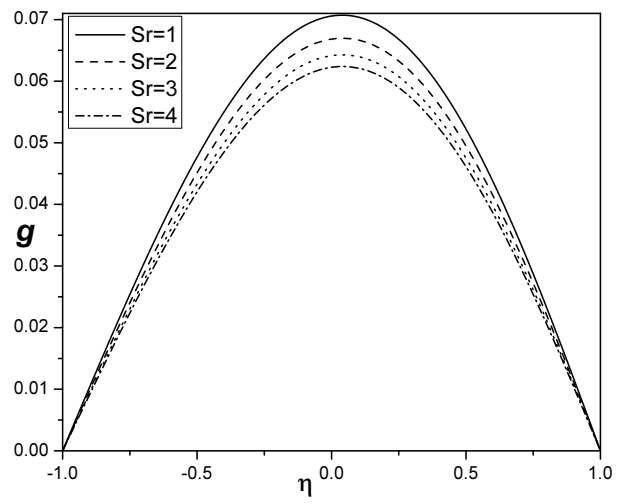

Figure 23: Effect of $S r$ on $g$ at $S=1, H a=2, \beta_{h}=2.0, D a=0.5$ 


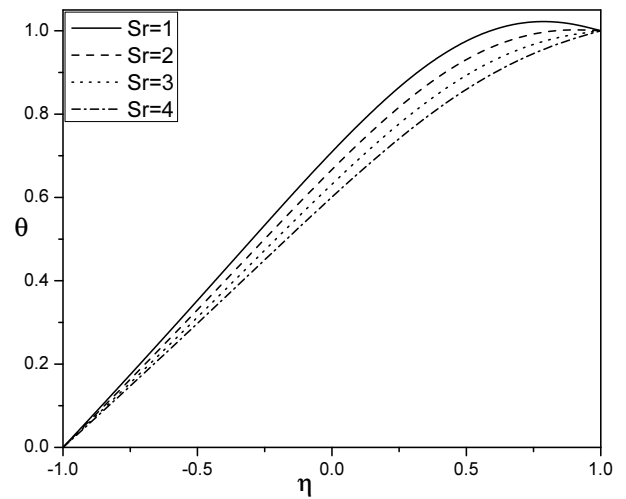

Figure 24: Effect of $S r$ on $\theta$ at $S=1, H a=2, \beta_{h}=2.0, D a=0.5$

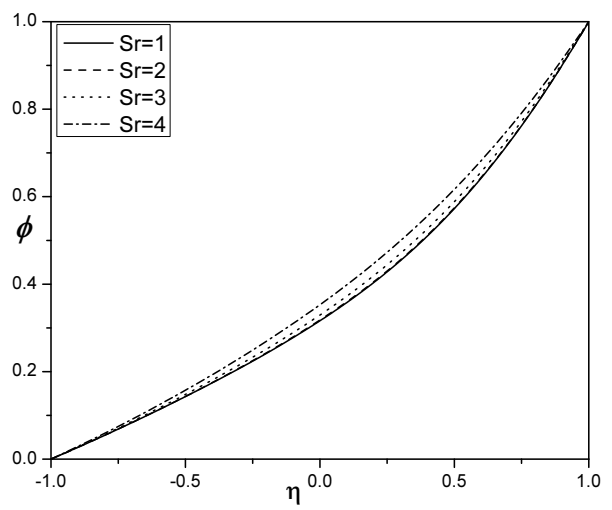

Figure 25: Effect of $S r$ on $\phi$ at $S=1, H a=2, \beta_{h}=2.0, D a=0.5$

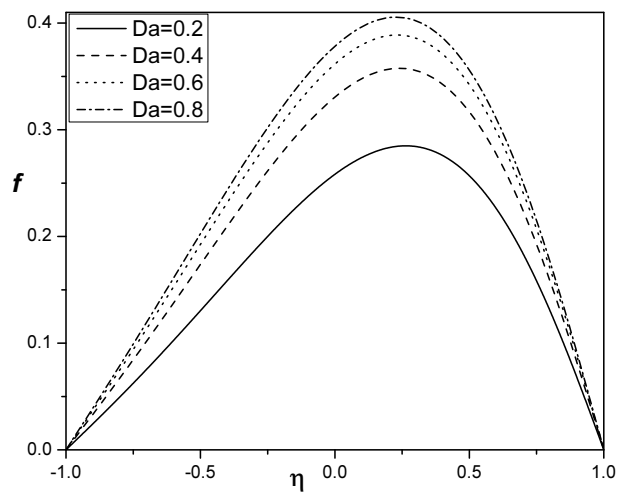

Figure 26: Effect of $D a$ on $f$ at $S=1, H a=2, \beta_{h}=2.0, S r=2$

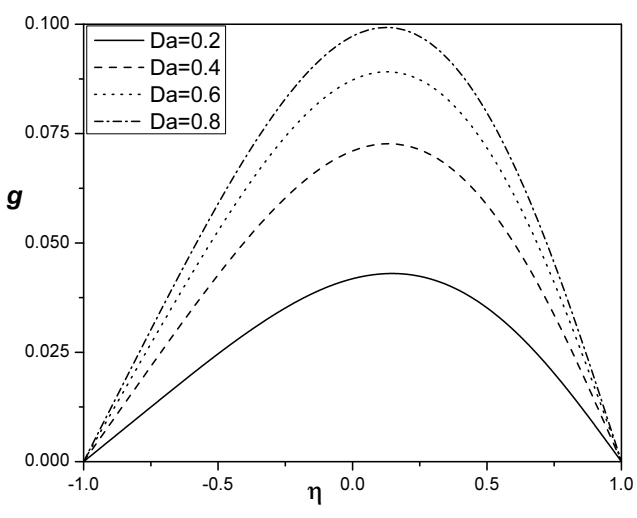

Figure 27: Effect of $\mathrm{Da}$ on $g$ at $S=1, \mathrm{Ha}=2, \beta_{h}=2.0, S r=2$

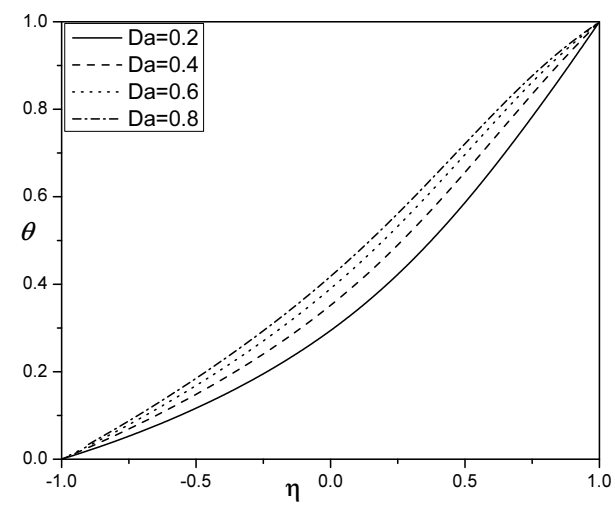

Figure 28: Effect of $D a$ on $\theta$ at $S=1, H a=2, \beta_{h}=2.0, S r=2$

appreciably influenced by the Soret number.

The nature of velocity components $(f(\eta)$ and $g(\eta))$, temperature $\theta(\eta)$ and concentration $\phi(\eta)$ with $D a$ are presented in Figs.26 to 29. We see that the dimensionless velocity component $f(\eta)$ increases with an increase in the darcy number. It can be seen from Fig. 27 that the induced velocity increases as $D a$ increases. This is due to the fact that the lower permeability enhances the flow, which leads to increases the velocities of the flow. The effect of darcy parameter on temperature profile is shown in Fig. 28. It is clear from this figure that the non-dimensional temperature profile increases with the increase of darcy parameter. Finally the influence of darcy number on concentration is shown in Fig. 29. It is observed from the figure that the dimensionless concentration decreases with an increase in darcy parameter. From this study we find that lesser the permeability of porous medium smaller the magnitude of the velocities, temperature and for further lowering of the permeability larger the magnitude of concentration in the entire flow region.

Variation of couple stress parameter $(S)$, thermal diffusion parameter $(\mathrm{Sr})$, magnetic parameter $\mathrm{Ha}$ together with the Hall number $\left(\beta_{h}\right)$ is presented in Table 4 with fixed values of other parameters. It is observed from this table that the skin friction coefficient increases with the increase of Soret parameter $(S r)$, where as heat transfer rate and mass transfer rates have reverse trend at both the plates, this is because of the applied magnetic field which is applied from one plate $\eta=-1$. It can be seen that the skin friction coefficient and heat transfer rate decreases at the initial plate and increases at the terminal plate where as the reverse trend 


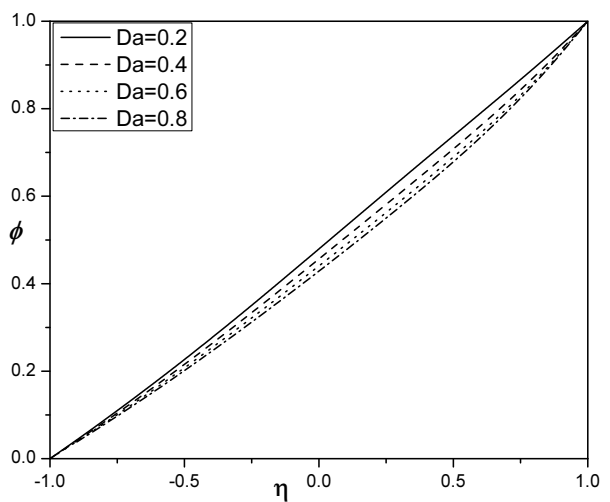

Figure 29: Effect of $D a$ on $\phi$ at $S=1, H a=2, \beta_{h}=2.0, S r=2$

Table 1: Total average residual errors at different order of approximations

\begin{tabular}{ll}
\hline Order & Error \\
\hline 10 & $4.11 \times 10^{-7}$ \\
14 & $1.93 \times 10^{-10}$ \\
16 & $1.39 \times 10^{-11}$ \\
18 & $1.91 \times 10^{-12}$ \\
20 & $1.68 \times 10^{-14}$ \\
30 & $4.19 \times 10^{-20}$ \\
\hline
\end{tabular}

is noticed on mass transfer rate with an increase in $\mathrm{Ha}$. As magnetic parameter increases, the resistive force slow downs the friction factor and heat transfer rate at $\eta=-1$. The effect of couple stress parameter $S$ on friction factor, heat and mass transfer rates are shown in Table 4. It is clear that the values of $f^{\prime}$ are increases with an increase in $S$. The heat and mass transfer rates are decreases at the initial plate and those are increases at the other plate with the enhance in $S$. Finally, for fixed values of $\mathrm{S}, \mathrm{Sr}$ and $\mathrm{Ha}$ the effect of hall parameter on the skin-friction coefficient, the rate of heat and mass transfers are shown in this table. The behavior of these parameters are self-evident from the Table 4 and hence are not discussed for brevity.

\section{CONCLUSIONS}

The present study investigates the steady manetohydrodynamic flow of couple stress fluid in a vertical channel saturated with porous medium in presence of Hall, joule heating and the soret effects. HAM is used to solve the final dimensionless governing equations. The significant findings are summarized as:

Table 2: Convergence of HAM solutions for different order of approximations

\begin{tabular}{lllll}
\hline$m$ & $f(0)$ & $g(0)$ & $\theta(0)$ & $\phi(0)$ \\
\hline 05 & 0.09613224020 & 0.01396554134 & 0.2009053728 & 0.4545198791 \\
10 & 0.09771396108 & 0.01381574081 & 0.1975840548 & 0.4540248490 \\
15 & 0.09776432443 & 0.01380893259 & 0.1975921270 & 0.4539927535 \\
20 & 0.09776431461 & 0.01380895941 & 0.1975939094 & 0.4539927247 \\
25 & 0.09776431461 & 0.01380895907 & 0.1975939006 & 0.4539927229 \\
30 & 0.09776431461 & 0.01380895907 & 0.1975939006 & 0.4539927229 \\
\hline
\end{tabular}

Table 3: Comparison of flow velocity $(f)$ for $H a=R=G r_{T} / R e=$ $G r_{C} / R e=1 / D a=0$

\begin{tabular}{|l|ll|ll|}
\hline \multirow{2}{*}{$\eta$} & \multicolumn{2}{|c|}{$S=0.5$} & \multicolumn{2}{c|}{$S=1$} \\
\cline { 2 - 5 } & Analytical & HAM & Analytical & HAM \\
\hline-1 & 0 & 0 & 0 & 0 \\
-0.5 & 0.2275 & 0.2275 & 0.1057 & 0.1057 \\
0 & 0.3164 & 0.3164 & 0.1480 & 0.1480 \\
0.5 & 0.2275 & 0.2275 & 0.1057 & 0.1057 \\
-1 & 0 & 0 & 0 & 0 \\
\hline
\end{tabular}

Table 4: Effects of skin friction coefficient, heat and mass transfer rates for various values of $\mathrm{Sr}, \mathrm{Ha}, \beta_{h}$ and $S$ when $\mathrm{Re}=2, N=5, \mathrm{Pr}=$ $0.7, B r=0.5, G r=0.5$ and $S c=0.22$

\begin{tabular}{llllllllll}
\hline$S r$ & $H a$ & $\beta_{h}$ & $S$ & $f^{\prime}(-1)$ & $f^{\prime}(1)$ & $N u_{1}$ & $N u_{1}$ & $S h_{1}$ & $S h_{2}$ \\
\hline 0.2 & 2 & 2 & 0.5 & 0.1046 & -0.3918 & -0.0366 & 0.0630 & -0.1003 & -1.4130 \\
0.6 & 2 & 2 & 0.5 & 0.1133 & -0.4061 & -0.0387 & 0.0661 & -0.1018 & -1.4055 \\
1 & 2 & 2 & 0.5 & 0.1219 & -0.4202 & -0.0407 & 0.0692 & -0.1035 & -1.3977 \\
1 & 1 & 2 & 0.5 & 0.1499 & -0.4655 & -0.0130 & 0.0215 & -0.1085 & -1.3746 \\
1 & 3 & 2 & 0.5 & 0.0793 & -0.3496 & -0.0474 & 0.1063 & -0.0928 & -1.4311 \\
1 & 5 & 2 & 0.5 & 0.0119 & -0.2130 & -0.0592 & 0.1116 & -0.0936 & -1.4721 \\
1 & 2 & 2 & 0.2 & 0.0295 & -0.7361 & -0.0220 & 0.1223 & -0.1035 & -1.3272 \\
1 & 2 & 2 & 0.4 & 0.1000 & -0.4983 & -0.0382 & 0.0843 & -0.1029 & -1.3792 \\
1 & 2 & 2 & 0.6 & 0.1332 & -0.3599 & -0.0398 & 0.0563 & -0.1049 & -1.4123 \\
1 & 2 & 1 & 0.5 & 0.0941 & -0.3722 & -0.0359 & 0.0636 & -0.0999 & -1.4172 \\
1 & 2 & 3 & 0.5 & 0.1369 & -0.4449 & -0.0354 & 0.0591 & -0.1059 & -1.3873 \\
1 & 2 & 5 & 0.5 & 0.1486 & -0.4638 & -0.0251 & 0.0415 & -0.1078 & -1.3792 \\
\hline
\end{tabular}

- Fluid flow velocity and the temperature profiles are disintegrate where as the flow in $z$-direction and the concentration profile increases with an increase in $H a$.

- As $\beta_{h}$ increases, the flow direction velocity and temperature profiles are increases but reverse trend can be observed in case of induced flow velocity and the concentration.

- It is noticed that the presence of couple stresses in the fluid decreases the velocity, temperature and increases the concentration of the fluid.

- The velocities and temperature are decreases, while mass transfer rate increases with the increase in the soret parameter.

- Increase in the darcy number increases the velocities and temperature but decreases the concentration.

\section{NOMENCLATURE}

A

$B_{0}$

$\mathrm{Br}$

C

$C_{P}$

$D$

$D a$

$f$

$g_{a}$

$G r_{C}$

$G r_{T}$

$\mathrm{Ha}$

$K_{f}$

$\mathrm{Nu}$

$\operatorname{Pr}$

$q_{m}$

$q_{w}$

$R$

$R e$

$S$
Constant pressure gradient.

Uniform magnetic field.

Brinkman number.

Concentration.

Specific heat.

Solutal diffusivity.

Darcy number.

Reduced stream function.

Acceleration due to gravity.

Thermal Grashof number.

Energy Grashof number.

Magnetic parameter.

Thermal conductivity.

Nusselt number.

Prandtl number.

Mass transfer from the plate.

Heat transfer from the plate.

Suction/induction parameter.

Reynolds number.

Couple stress fluid parameter. 
Sc Schmidt number.

Sh Local Sherwood number.

Sr Soret Parameter.

$T \quad$ Temperature.

$T_{m} \quad$ Mean fluid temperature.

$u_{0} \quad$ Entrance flow velocity.

$u, v \quad$ Velocities in $x$ and $y$ directions.

$x, y \quad$ Coordinates along and normal to the plate.

$\beta_{T} \quad$ Thermal expansion coefficient.

$\beta_{C} \quad$ Solutal expansion coefficient.

$\eta \quad$ Pseudo-similarity variable.

$\eta_{1} \quad$ The coupling material constant.

$\mu \quad$ Dynamic viscosity.

$\nu \quad$ Kinematic viscosity.

$\phi \quad$ non-Dimensional concentration.

$\rho \quad$ fluid Density.

$\theta \quad$ non-Dimensional temperature.

\section{SUPERSCRIPT}

Differentiation with respect to $\eta$.

\section{REFERENCES}

Afify, A .A., 2 009, " Similarity S olution i n M HD: E ffects o f T hermal Diffusion and Diffusion Thermo on Free Convective Heat and Mass Transfer over a Stretching Surface Considering Suction or Injection," Communications in Nonlinear Science and Numerical Simulation, 14(5), 2202-2214. http://dx.doi.org/10.1016/j.cnsns.2008.07.001

Boulama, K., and Galanis, N., 2004, "Analytical Solution for Fully Developed Mixed Convection Between Parallel Vertical Plates With Heat and Mass Transfer," Journal of Heat Transfer, 126(3), 381.

http://dx.doi.org/10.1115/1.1737774

Fattahi, E., Farhadi, M., Sedighi, K., and Nemati, H., 2012, "Lattice Boltzmann Simulation of Natural Convection Heat Transfer in Nanofluids," International Journal of Thermal Sciences, 52, 137-144, doi:http://dx.doi.org/10.1016/j.ijthermalsci.2011.09.001

Hasnain, J., Abbas, Z., and Sajid, M., 2015, "Effects of Porosity and Mixed Convection on MHD Two Phase Fluid Flow in an Inclined Channel," PloS one, 10(3), e0119913,

http://dx.doi.org/10.1371/journal.pone.0119913

Huelsz, G., and Rechtman, R., 2013, "Heat Transfer Due to Natural Convection in an Inclined Square Cavity Using the Lattice Boltzmann Equation Method," International Journal of Thermal Sciences, 65, 111-119, http://dx.doi.org/10.1016/j.ijthermalsci.2012.09.009

Liao, S., 2003, Beyond perturbation. Introduction to Homotopy Analysis Method, Chapman and Hall/CRC Press, Boca Raton.

Liao, S., 2010, "An Optimal Homotopy-Analysis Approach for Strongly Nonlinear Differential Equations," Communications in Nonlinear Science and Numerical Simulation, 15(8), 2003-2016.

http://dx.doi.org/10.1016/j.cnsns.2009.09.002
Liao, S., 2014, Advances in the Homotopy Analysis Method, World Scientific Publishing Co. Pte. Ltd., Singapore.

Mahmoud, M.A.A., and Megahed, A.M., 2013, "Thermal Radiation Effect on Mixed Convection Heat and Mass Transfer of a Non-Newtonian Fluid over a Vertical Surface Embedded in a Porous Medium in the Presence of Thermal Diffusion and Diffusion-Thermo Effects," Journal of Applied Mechanics and Technical Physics, 54(1), 90-99.

http://dx.doi.org/10.1134/S0021894413010112.

Makinde, O., and Eegunjobi, A., 2013, "Entropy Generation in a Couple Stress Fluid Flow Through a Vertical Channel Filled with Saturated Porous Media," Entropy, 15(11), 4589-4606. http://dx.doi.org/10.3390/e15114589.

Martyushev, S.G., and Sheremet, M.A., 2014, "Conjugate Natural Convection Combined with Surface Thermal Radiation in an Air Filled Cavity with Internal Heat Source," International Journal of Thermal Sciences, 76, 51-67. http://dx.doi.org/10.1016/j.ijthermalsci.2013.08.012.

Rahman, M.M., Saidur, R., and Rahim, N.A., 2011, "Conjugated Effect of Joule Heating and Magneto-Hydrodynamic on Double-Diffusive Mixed Convection in a Horizontal Channel with an Open Cavity," International Journal of Heat and Mass Transfer, 54(15-16), 3201-3213. http://dx.doi.org/10.1016/j.ijheatmasstransfer.2011.04.010.

Srinivasacharya, D., and Kaladhar, K., 2012, "Mixed Convection Flow of Couple Stress Fluid Between Parallel Vertical Plates with Hall and Ion-Slip Effects," Communications in Nonlinear Science and Numerical Simulation, 17(6), 2447-2462, http://dx.doi.org/10.1016/j.cnsns.2011.10.006

Srinivasacharya, D., Mallikarjuna, B., and Bhuvanavijaya, R., 2015, "Soret and Dufour Effects on Mixed Convection Along a Vertical Wavy Surface in a Porous Medium with Variable Properties," Ain Shams Engineering Journal, 6(2), 553564.

http://dx.doi.org/10.1016/j.asej.2014.11.007

Stokes, V.K., 1966, "Couple Stresses in Fluids," Physics of Fluids, 9, 1709, http://dx.doi.org/10.1063/1.1761925

Stokes, V.K., 1984, Theories of Fluids with Microstructure, Springer Berlin Hei-delberg, Berlin, Heidelberg.

http://dx.doi.org/10.1007/978-3-642-82351-0

Turkyilmazoglu, M., 2011a, "Convergence of the Homotopy Perturbation Method," International Journal of Nonlinear Sciences and Numerical Simulation, 12(1-8).

http://dx.doi.org/10.1515/ijnsns.2011.020

Turkyilmazoglu, M., 2011b, "Some Issues on HPM and HAM Methods: A Convergence Scheme," Mathematical and Computer Modelling, 53(9-10), 1929-1936, http://dx.doi.org/10.1016/j.mcm.2011.01.022

Turkyilmazoglu, M., 2014, "Exact Solutions for Two-Dimensional Laminar Flow Over a Continuously Stretching or Shrinking Sheet in an Electrically Conducting Quiescent Couple Stress Fluid," International Journal of Heat and Mass Transfer, 72, 1-8.

http://dx.doi.org/10.1016/j.ijheatmasstransfer.2014.01.009

Zhang, J.K., Li, B.W., and Chen, Y.Y., 2015, "The Joule Heating Effects on Natural Convection of Participating Magnetohydrodynamics Under Different Levels of Thermal Radiation in a Cavity," Journal of Heat Transfer, 137(5), 52502. http://dx.doi.org/10.1115/1.4029681 